\title{
Towards easier realization of time-delayed feedback control of odd-number orbits
}

\author{
V. Flunkert* and E. Schöll \\ Institut für Theoretische Physik, Technische Universität Berlin, Hardenbergstraße 36, D-10623 Berlin, Germany
}

(Received 1 April 2011; published 19 July 2011)

\begin{abstract}
We develop generalized time-delayed feedback schemes for the stabilization of periodic orbits with an odd number of positive Floquet exponents, which are particularly well suited for experimental realization. We construct the parameter regimes of successful control and validate these by numerical simulations and numerical continuation methods. In particular, it is shown how periodic orbits can be stabilized with symmetric feedback matrices by introducing an additional latency time in the control loop. Finally, we show using normal form analysis and numerical simulations how our results could be implemented in a laser setup using optoelectronic feedback.
\end{abstract}

DOI: 10.1103/PhysRevE.84.016214

PACS number(s): $05.45 . \mathrm{Gg}, 02.30 . \mathrm{Ks}$, 42.55.Px, 02.30.Oz

\section{INTRODUCTION}

Time-delayed feedback control has been proposed by Pyragas [1] as a method of stabilizing unstable periodic orbits (UPOs) in dynamical systems. Although the control scheme is very simple, it has an intriguing feature: If the target orbit is stabilized by the control, then the control force vanishes on the target orbit, and therefore the orbit is stabilized but otherwise unchanged. This remarkable property has drawn a lot of attention [2-5] to the Pyragas control for two reasons: First, the noninvasive stabilization of unstable states makes it possible to study these states in experiments, i.e., unravel dynamical behavior that is usually hidden [3,6,7]. Second, noninvasive control means that the system is subject to small control signals only. This is important whenever there are limited resources, e.g., constraints due to a finite fuel tank or limits on power consumptions, or when the system to be controlled is fragile and one wants to avoid strong forcing, e.g., in neural applications. Although classical difference feedback control is noninvasive too, the advantage of Pyragas control is that the exact location of the target orbit or fixed point does not need to be known. Additionally, Pyragas control is particularly well suited for implementation in optical systems via Fabry-Perot resonators.

For a dynamical system $\dot{X}=f(X)$ with $X \in \mathbb{R}^{n}$ and some nonlinear function $f$, linear Pyragas control is introduced as

$$
\dot{X}(t)=f(X(t))+K[X(t-\tau)-X(t)],
$$

where $K$ is a $n \times n$ feedback gain matrix, and $\tau$ is the delay time. The control scheme utilizes the system history to generate a control signal, which is fed back to the system in a closed-loop fashion. The advantages of such a closed-loop control scheme are apparent: There is no need for real time computation of control signals, and no reference or target state needs to be known. Instead, the system generates its own control signal, and by choosing the parameters of the control loop, e.g., delay time and feedback strengths, appropriately, the system operates in the desired regime. In many experimental situations it is relatively easy to introduce this kind of control. The delay time $\tau$ has to be chosen as a multiple of the target orbit's period in order for the control to be noninvasive. It is

\footnotetext{
*flunkert@itp.tu-berlin.de
}

then necessary to tune the control parameters, i.e., the matrix $K$, such that the orbit is stabilized.

For more than a decade it was believed [8-12] that one of the most common types of UPOs, so-called odd-number orbits, could not be stabilized with the Pyragas method. These odd-number orbits have an odd number of real unstable Floquet multipliers and occur, for instance, in many of the most fundamental bifurcations, such as subcritical Hopf bifurcations and fold bifurcations of UPOs.

Recently it was shown that contrary to this common belief, odd-number orbits can be stabilized, refuting the so-called odd-number theorem [13]. This surprising turn resulted in a renewed interest in Pyragas control in recent years [14-24].

In this work we extend and generalize the original counterexample to the odd-number theorem and construct a class of feedback schemes that are better suited for experimental realizations. The paper is organized as follows: In Sec. II, we briefly discuss the main ideas of the original counterexample. Based on this discussion, we develop new control schemes in Sec. III and validate these schemes with numerical methods. Section IV then further extends the control to stabilization with symmetric feedback matrices using an additional latency time in the control loop. To demonstrate our proposed control schemes, we apply them to a laser model in Sec. V using normal form analysis and numerical simulations. Finally, we summarize our results in Sec. VI.

\section{REVIEW OF THE COUNTEREXAMPLE}

In this section we briefly discuss the counterexample to the alleged odd-number theorem introduced in Ref. [13]. It consists of a subcritical Hopf bifurcation with a time-delayed feedback term of Pyragas type:

$$
\frac{d}{d t} z=\left[\mu+i+(1+i \gamma)|z|^{2}\right] z+b[z(t-\tau)-z(t)] .
$$

Here $z \in \mathbb{C}$ is the complex dynamical variable, $\mu \in \mathbb{R}$ is the normal form bifurcation parameter, the linear angular frequency has been scaled to $\omega=1$, and $\gamma \in \mathbb{R}$ is the nonisochronicity or shear parameter, which couples the phase and the amplitude of the oscillator. The Pyragas control term is given by $b[z(t-\tau)-z(t)]$, where $b \in \mathbb{C}$ is the complex feedback strength. For brevity, we omit the time argument of dynamical variables where the meaning is clear. 


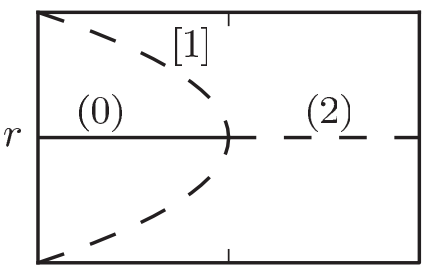

(a)

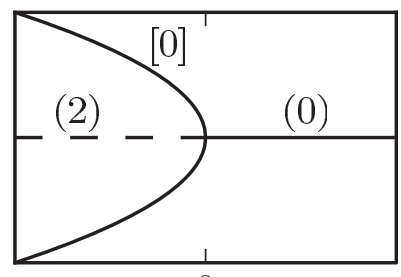

(b)

FIG. 1. Conversion of stability. (a) Subcritical Hopf bifurcation according to Eq. (1) without control. The numbers in parentheses denote the number of unstable dimensions of the fixed point. The numbers in square brackets denote the number of unstable Floquet multipliers of the periodic orbit. (b) If the stability of the fixed point has been reversed and the position of the periodic orbit remains unchanged, the periodic orbit must have become stable.

For $\mu<0$ an UPO with amplitude $|z|=\sqrt{-\mu}$ and period $T=2 \pi /(1-\gamma \mu)$ exists [see Fig. 1(a)]. According to Pyragas control the delay is chosen as an integer multiple of the period $\tau=n T, n \in \mathbb{N}$, such that the control is noninvasive in case of successful stabilization. Since Eq. (1) describes an autonomous system, the UPO has a Floquet multiplier equal to 1 corresponding to the Goldstone mode. The other Floquet multiplier is real and has magnitude larger than 1, because the periodic orbit (PO) is unstable. The UPO is thus of odd-number type, and it would not be possible to stabilize it with the feedback of Eq. (1) if the odd-number theorem were correct.

One could try to analyze the PO's stability using Floquet theory and try to find appropriate control forces $b$. However, the Floquet problem for delay differential equations is very difficult to treat analytically. The idea in Ref. [13] to approach the problem, nevertheless, is to construct conditions such that the fixed point (FP) $z=0$ is unstable (with two unstable dimensions) for $\mu<0$ and stable for $\mu>0$ as is depicted in Fig. 1(b). If one succeeds while preserving the location of the $\mathrm{PO}$, as is the case for noninvasive time-delayed feedback control, then the subcritical Hopf bifurcation must have become supercritical, and the PO is stable in the vicinity of $\mu=0$. This was corroborated by showing that the UPO can gain stability through collision with a stable delay-induced periodic orbit in a transcritical bifurcation, exchanging stability.

Thus by constructing conditions such that the FP changes stability in the proper way, we can ensure stabilization of the PO close to the bifurcation $\mu=0$. This is accomplished with complex feedback gains $b=b_{0} e^{i \beta}$ with nonzero feedback phase $\beta$ in Ref. [13] refuting the alleged odd-number theorem.

In the next section we will consider an alternative feedback, which is closer to experimental situations, and use the construction of the original counterexample to find parameters for which stabilization is successful.

\section{EXPERIMENTALLY RELEVANT FEEDBACK MATRICES}

As shown theoretically by the counterexample discussed in the last section, the odd-number limitation does not hold, and this has also been verified by recent experiments with electronic circuits [23] and lasers [24].

However, the feedback scheme is not particularly well suited for implementation in usual experimental situations. One reason why the counterexample is not immediately applicable is the special choice of the gain matrix. This gain matrix conserves the $S^{1}$ symmetry of the normal form, but in order to realize this control matrix experimentally, one needs to have access to two dynamical variables in the rotational plane of the orbit, process these to generate the rotation phase $\beta$, and feed the control signal back into the corresponding two dynamic degrees of freedom. This may be possible in certain situations, for instance, when stabilizing an unstable mode of a laser, where the optical phase can naturally introduce a rotation $[18,24]$ or in particular electronic setups [23]. But what happens, for example, if we can control only one dynamical variable? We will give some answers to this question in the following.

Consider a dynamical system with a bifurcation parameter $\mu$ that undergoes a subcritical Hopf bifurcation at $\mu=0$ with the UPO lying (without loss of generality) on the $\mu<0$ side. The center manifold theorem implies that close to the bifurcation the uncontrolled system equations can be transformed to the real normal form

$$
\frac{d}{d t}\left(\begin{array}{l}
x \\
y
\end{array}\right)=\left[\begin{array}{cc}
d \mu+a r^{2} & -\left(\omega+c \mu+b r^{2}\right) \\
\omega+c \mu+b r^{2} & d \mu+a r^{2}
\end{array}\right]\left(\begin{array}{l}
x \\
y
\end{array}\right),
$$

with $r^{2}=x^{2}+y^{2}$ and real parameters $a, b, c, d$. We choose $d>0$ (the FP is stable or unstable for $\mu<0$ or $\mu>0$, respectively) and $a>0$ (the UPO lies on the $\mu<0$ side). For simplicity we will assume $\omega>0$. The case $\omega<0$ is easily recovered by exchanging the variables $x \longleftrightarrow y$.

Although these equations can be simplified further to the complex form of Eq. (1) by rescaling the bifurcation parameter, the time, and the dynamical variables, we keep this real form of the equations to allow for easier comparison with experimental situations. In particular we calculate the normal form coefficients $\omega, a, b, c$, and $d$ for a laser model in Sec. V.

In polar coordinates $r, \theta$ the equations are given by

$$
\begin{aligned}
& \frac{d}{d t} r=\left(d \mu+a r^{2}\right) r, \\
& \frac{d}{d t} \theta=\left(\omega+c \mu+b r^{2}\right),
\end{aligned}
$$

and the radius $r$ and period $T$ of the UPO can be read off

$$
\begin{gathered}
r=\sqrt{-\frac{d}{a} \mu}, \\
T=\frac{2 \pi}{\left|\omega+c \mu+b r^{2}\right|}=\frac{2 \pi}{\left|\omega+\left(c-\frac{b d}{a}\right) \mu\right|} .
\end{gathered}
$$

Let us now consider linear Pyragas feedback with a general coupling matrix $K_{i j}$ :

$$
\begin{aligned}
\frac{d}{d t}\left(\begin{array}{l}
x \\
y
\end{array}\right)= & {\left[\begin{array}{cc}
d \mu+a r^{2} & -\left(\omega+c \mu+b r^{2}\right) \\
\omega+c \mu+b r^{2} & d \mu+a r^{2}
\end{array}\right]\left(\begin{array}{l}
x \\
y
\end{array}\right) } \\
& +\left[\begin{array}{ll}
K_{11} & K_{12} \\
K_{21} & K_{22}
\end{array}\right]\left(\begin{array}{l}
x(t-\tau)-x(t) \\
y(t-\tau)-y(t)
\end{array}\right) .
\end{aligned}
$$

We follow the idea of Ref. [13] and analyze the stability of the FP. Note that this refers only to local stability criteria. Making the ansatz $(x, y)=u e^{\eta t}$, where $u$ is a constant vector, 
we obtain the transcendental characteristic equation $\chi(\eta)=0$ for the eigenvalues of the FP:

$\chi(\eta):=\operatorname{det}\left[\begin{array}{cc}d \mu-\eta+K_{11} F(\eta) & -\omega-c \mu+K_{12} F(\eta), \\ \omega+c \mu+K_{21} F(\eta) & d \mu-\eta+K_{22} F(\eta) .\end{array}\right]$,

where $F(\eta)=\left(e^{-\eta \tau}-1\right)$. Calculating the determinant yields

$$
\begin{aligned}
\chi(\eta)= & (d \mu-\eta)^{2}+\operatorname{tr} K(d \mu-\eta) F(\eta)+\operatorname{det} K F(\eta)^{2} \\
& +(\omega+c \mu)^{2}+\kappa(\omega+c \mu) F(\eta) .
\end{aligned}
$$

Here we have introduced the parameter $\kappa:=K_{21}-K_{12}$, which is a measure for the asymmetry of the feedback matrix ( $\kappa=0$ for symmetric matrices) and will play a crucial role in the following analysis. Note that when we recover the case of negative $\omega$ by exchanging $x$ and $y$ as discussed above, $\kappa$ changes sign in the characteristic equation $\kappa \rightarrow-\kappa$.

For the general case of an arbitrary coupling matrix $K$ the characteristic equation cannot be treated with the method of Ref. [13]. However, the practically most relevant case, where one measures a single variable $u$ and applies the control to a single variable $v$, is treatable. After the center manifold reduction (and normal form transformation) $u$ and $v$ are functions of $x$ and $y$, which we expand to the leading linear order

$$
\begin{gathered}
u=u(x, y)=u_{1} x+u_{2} y+\cdots, \\
v=v(x, y)=v_{1} x+v_{2} y+\cdots .
\end{gathered}
$$

Here we have omitted constant terms, since they would disappear in the Pyragas feedback. The measured signal $m$ is given by the projection

$$
m(t)=\left(\begin{array}{l}
u_{1} \\
u_{2}
\end{array}\right) \cdot\left(\begin{array}{l}
x(t) \\
y(t)
\end{array}\right)
$$

and our control signal acts as

$$
\begin{aligned}
& \frac{d}{d t} x=\cdots+v_{1}[m(t-\tau)-m(t)], \\
& \frac{d}{d t} y=\cdots+v_{2}[m(t-\tau)-m(t)]
\end{aligned}
$$

on the dynamical equations. This leads to the following gain matrix:

$$
K=\left[\begin{array}{ll}
v_{1} u_{1} & v_{1} u_{2} \\
v_{2} u_{1} & v_{2} u_{2}
\end{array}\right]
$$

which has vanishing determinant, reflecting the fact that the control acts only in one direction. With det $K=0$ the characteristic equation (4) simplifies to

$$
\begin{aligned}
0= & (d \mu-\eta)^{2}+\operatorname{tr} K(d \mu-\eta) F(\eta) \\
& +(\omega+c \mu)^{2}+\kappa(\omega+c \mu) F(\eta) .
\end{aligned}
$$

For this simpler equation it is now possible to carry out the analysis. Since the system without control is invariant under rotations, we can without loss of generality choose the $v$ vector to point in the $x$ direction. With this choice the control matrix is given by

$$
K=\left[\begin{array}{cc}
\operatorname{tr} K & -\kappa \\
0 & 0
\end{array}\right]
$$

In the following we have to distinguish different cases, due to different sign combinations of the various parameters.
In particular we have to distinguish between the increasing period (IP) case

$$
-(c-b d / a)<0,
$$

where the period of the UPO increases with increasing distance from the bifurcation and the decreasing period (DP) case

$$
-(c-b d / a)>0,
$$

where it decreases with increasing distance from the bifurcation. Note that this distinction is not exactly the same as soft $(b<0)$ and hard $(b>0)$ spring, which was discussed in Ref. [21], because the parameter $c$, which changes the period with the bifurcation parameter, can overrule the other term $b d / a$, which changes the period with the amplitude of the oscillations.

\section{A. Stabilization conditions}

The domain of $\kappa$ depends on whether the period increases or decreases with increasing distance from the bifurcation

$$
\begin{array}{llll}
\kappa>0, & \text { if } & c-b d / a>0 & \text { (IP case) } \\
\kappa<0, & \text { if } & c-b d / a<0 & \text { (DP case) } .
\end{array}
$$

In both cases the domain of control is close to the bifurcation (lim $\mu \rightarrow 0$ ) bounded by

$$
\begin{gathered}
-\omega \frac{2 n+1}{2 n^{2}} \leqslant \kappa \leqslant \omega \frac{2 n-1}{2 n^{2}}, \\
\operatorname{tr} K \geqslant \frac{\kappa \omega}{\sqrt{\omega^{2}-2 \kappa \omega}} \cot (\pi n \sqrt{1-2 \kappa / \omega}), \\
\operatorname{tr} K<-\frac{b}{a} \kappa-\frac{\omega}{n \pi} .
\end{gathered}
$$

Figures 2 and 3 depict the control domain for the two cases. The dotted blue line in Fig. 2 marks the stability domain of the Pyragas orbit for a finite value of $\mu=-0.005$ calculated

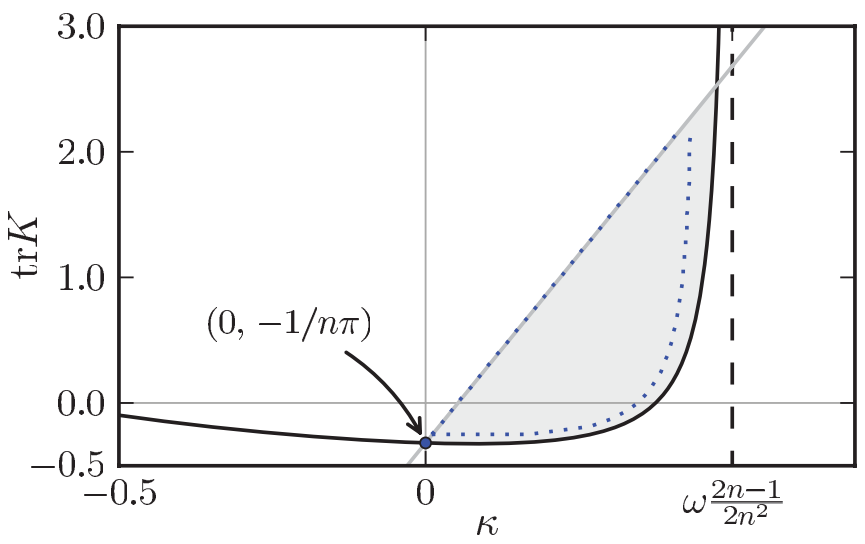

FIG. 2. (Color online) Control domain (shaded) for the case of increasing period with $\kappa>0$. The black curve and the tilted straight line correspond to Eqs. (6b) and (6c), respectively. The dashed vertical line marks the boundary corresponding to the right boundary in Eq. (6a). The tilted line has a slope of $-b / a$. The dotted line shows the exact stability domain of the target orbit for $\mu=0.005$ and is calculated with the continuation software KNUT. Parameters: $\omega=1$, $n=1, b / a=-6$. 


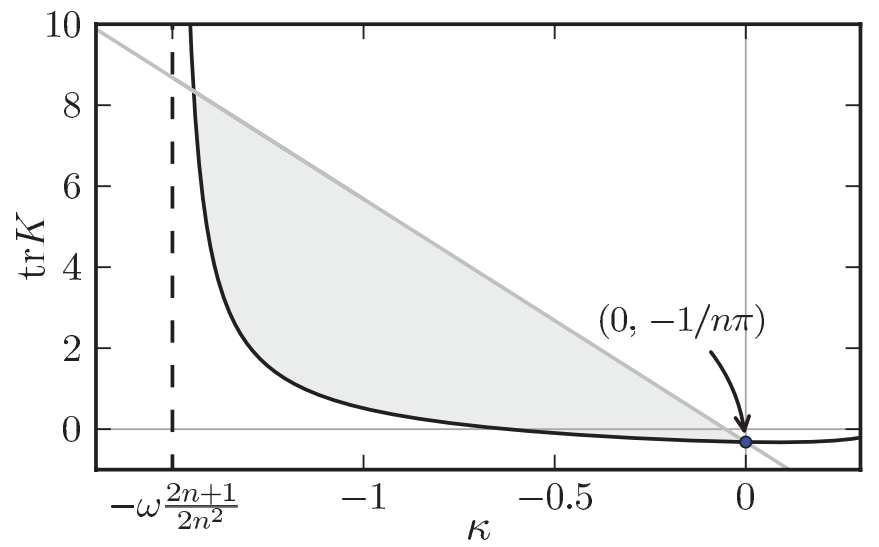

FIG. 3. (Color online) Control domain (shaded) for the case of decreasing period with $\kappa<0$. The dashed curve and the tilted straight line correspond to Eqs. (6b) and (6c), respectively. The dashed line marks the boundary corresponding to the left boundary in Eq. (6a). The tilted line has a slope of $-b / a$. Parameters: $\omega=1, n=1$, $b / a=6$.

with the continuation software KNUT [25]. For small values of $\mu$ the domain is well approximated by the analytic result (shaded area).

As the two boundary curves Eqs. (6b) and (6c) intersect in the point $(\kappa, \operatorname{tr} K)=(0,-\omega / n \pi)$ stabilization is not possible with symmetric feedback matrices, because these have $\kappa=0$. It is easy to check that even in the case of more than one input variable (det $K \neq 0$ ) control is not possible with $\kappa=0$, because the Hopf curves are tangent to the $\tau$ axis at the Pyragas points and do not cross the $\tau$ axis at these points. This includes the result of Ref. [13], where feedback with zero rotation angle does not allow for control. This imposes a severe limitation for the experimental applicability, because the case $\kappa=0$ occurs when one can measure only a single variable and apply the control signal to the dynamic equation of the same variable. Due to the importance of this situation we will discuss a method to overcome this restriction in the next section.

We can find conditions which ensure a nonempty control domain. The right-hand side of Eq. (6b) is a convex function of $\kappa$ and has a slope of $-1 /(2 \pi n)$ at the intersection point with the other boundary [Eq. (6c)]. This other boundary (straight line) has a slope of $-b / a$. The following conditions thus lead to a nonempty control domain

$$
\begin{aligned}
& -\frac{b}{a}>-\frac{1}{2 \pi n} \quad \text { for the IP case } \quad(\kappa>0), \\
& -\frac{b}{a}<-\frac{1}{2 \pi n} \quad \text { for the DP case } \quad(\kappa<0) .
\end{aligned}
$$

From these two equations we can see that in the DP case stabilization is possible only for hard springs $(b>0)$, whereas in the IP case we are able to stabilize soft springs $(b<0)$ as well as weakly hard springs $[0<b<1 /(2 \pi n)]$.

\section{B. Derivation of stabilization results}

To derive the stabilization conditions, we construct parameters for which the FP is unstable with two unstable directions on the $\mu<0$ side and stable for $\mu>0$ as discussed in the previous section.

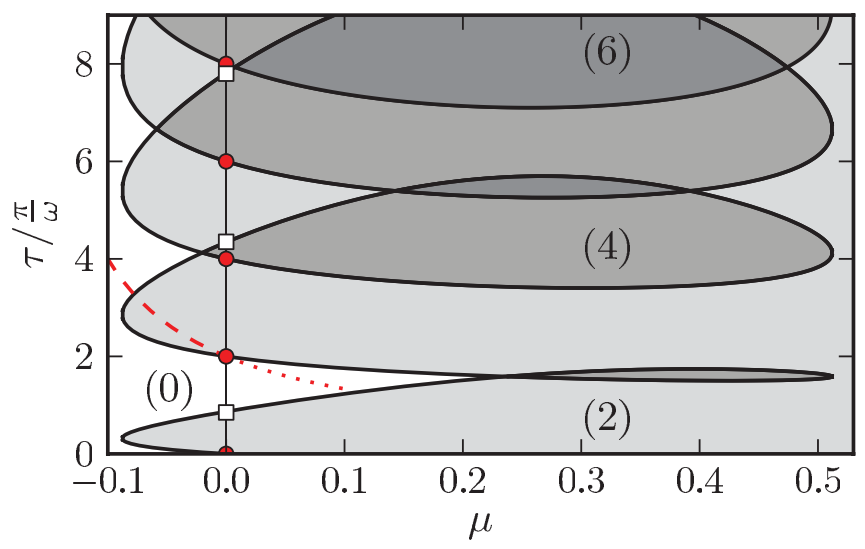

FIG. 4. (Color online) Hopf bifurcation line (solid) and Pyragas curve (dashed and dotted) in the $(\mu, \tau)$ plane. The numbers in parentheses and the corresponding shading denote the number of unstable dimensions of the fixed point. The red circles and the white squares depict points of the Hopf $A$ and $B$ series, respectively.

The basic idea to construct such parameters is as follows: For $\tau=0$ the control term vanishes, and there is thus a Hopf bifurcation at $(\mu, \tau)=(0,0)$. This Hopf bifurcation can be continued in the $(\mu, \tau)$ plane for a given feedback matrix $K$ as shown in Fig. 4. On the other hand, Pyragas control chooses the delay time to be an integer multiple of the orbit's period $\tau=n T$, which results in the Pyragas curve in the $(\mu, \tau)$ plane as shown by the dashed line in Fig. 4 for $n=1$. The dotted line shows an (arbitrary) differentiable extension of the Pyragas curve into the $\mu>0$ line, where the orbit does not exist and the period is thus not defined. In the situation sketched in Fig. 4, the Hopf and the Pyragas curve are oriented such that along the Pyragas curve the desired exchange of stability takes place: For $\mu<0$ the FP is twofold unstable and for $\mu>0$ the FP is stable. How can we find parameters for the control that lead to this geometric situation?

As a result of the noninvasiveness of the control, the Hopf curve intersects the $\mu=0$ line at integer multiples of $2 \pi / \omega$, as we will see below. We call these Hopf points on the $\tau$ axis the Hopf A series (red circles in Fig. 4). Note that the Pyragas curves emanate from these points. We will hence also refer to these points as Pyragas points. Additionally, there are other crossing points, which we will call Hopf $B$ series (white squares in Fig. 4). Using these two series of Hopf points and the slopes of the Hopf and Pyragas curve at these points, we can construct parameters (feedback gain) such that there is a change from (0) to (2) along the Pyragas curve, where the numbers in parentheses denote the total number of eigenvalues with $\operatorname{Re}(\eta)>0$ of the FP, i.e., the number of unstable dimensions; see Fig. 4.

We thus have to find the following:

(1) The location of the Hopf points on the $\tau$ axis (Hopf $A$ and $B$ points);

(2) The crossing direction of the Hopf eigenvalues at these Hopf points when going up the $\tau$ axis, which determines whether the unstable dimension of the FP increases or decreases by 2 ;

(3) The slope of the Hopf curve and the Pyragas curve at the Hopf $A$ points. 
In the following we will refer to these stabilization ingredients and the reader should keep Fig. 4 in mind during the discussion.

(1) Location of Hopf points: To find the location of the Hopf points on the $\tau$ axis, we insert $\eta=i \Omega$ into Eq. (5), set $\mu=0$, and split the equation into real and imaginary parts:

$$
\begin{aligned}
& 0=-\Omega^{2}-\kappa \omega+\omega^{2}+\kappa \omega \cos (\Omega \tau)-\Omega \operatorname{tr} K \sin (\Omega \tau), \\
& 0=\Omega \operatorname{tr} K(1-\cos (\Omega \tau))-\kappa \omega \sin (\Omega \tau) .
\end{aligned}
$$

In the following we will for simplicity consider $\Omega$ to be positive. The complex conjugate solution is simply $\eta=-i \Omega$. Writing Eqs. (7) as

$$
\left(\begin{array}{c}
\Omega^{2}-\omega^{2}+\kappa \omega \\
-\Omega \operatorname{tr} K
\end{array}\right)=\left[\begin{array}{cc}
\cos \xi & \sin \xi \\
-\sin \xi & \cos \xi
\end{array}\right]\left(\begin{array}{c}
\kappa \omega \\
-\Omega \operatorname{tr} K
\end{array}\right),
$$

with $\xi=\Omega \tau$, it is obvious that there can be a solution only if the two vectors have the same length

$$
\left(\Omega^{2}-\omega^{2}+\kappa \omega\right)^{2}+\Omega^{2} \operatorname{tr} K^{2}=\kappa^{2} \omega^{2}+\Omega^{2} \operatorname{tr} K^{2},
$$

since the rotation matrix leaves the length of vectors invariant. This gives the values for $\Omega^{2}$ on the $\tau$ axis

$$
\Omega^{2}=\omega^{2}, \quad \Omega^{2}=\omega^{2}-2 \kappa \omega .
$$

With these $\Omega$ values we can calculate the rotation angle $\xi$ and the delay time $\tau$ values. In particular for $\Omega^{2}=\omega^{2}$ we recover the Pyragas points (alias $A$ series) on the $\tau$ axis

$$
\tau_{n}^{A}=\frac{2 \pi n}{\omega}, \quad \Omega^{A}=\omega .
$$

Inserting $\Omega=\Omega^{B}:=\sqrt{\omega^{2}-2 \kappa \omega}$ gives the $B$ series. Note that $\kappa<\omega / 2$ is necessary in order for Hopf $B$ points to exist. When calculating $\xi$ (and $\tau$ ) for the Hopf $B$ points we have to take into account the different possible signs of $\kappa$ and $\operatorname{tr} K$ :

$$
\tau_{n}^{B}=\left\{\begin{array}{cc}
\frac{1}{\Omega^{B}}[2 \pi n+\varphi], & \text { if } \quad \kappa \cdot \operatorname{tr} K \geqslant 0, \\
\frac{1}{\Omega^{B}}[2 \pi n+(2 \pi-\varphi)], & \text { if } \quad \kappa \cdot \operatorname{tr} K<0,
\end{array}\right.
$$

with

$$
\varphi=\arccos \left[\frac{\operatorname{tr} K^{2}\left(\omega^{2}-2 \kappa \omega\right)-\omega^{2} \kappa^{2}}{\operatorname{tr} K^{2}\left(\omega^{2}-2 \kappa \omega\right)+\omega^{2} \kappa^{2}}\right] .
$$

The index is chosen such that $n=0$ labels the first point in the series, i. e., $n=0$ is the lowest integer with $\tau_{n}^{B}>0$. The Hopf $B$ series is spread equidistantly on the $\tau$ axis with a distance

$$
\Delta \tau^{B}=\tau_{n+1}^{B}-\tau_{n}^{B}=\frac{2 \pi}{\sqrt{\omega^{2}-2 \kappa \omega}} .
$$

(2) Crossing direction of Hopf eigenvalue pair: The crossing direction of the Hopf eigenvalues for Hopf points on the $\tau$ axis is given by

$$
\begin{aligned}
\operatorname{sgn} \operatorname{Re}\left(\partial_{\tau} \eta\right)= & \operatorname{sgn}\left[-\Omega^{2} \operatorname{tr} K^{2}+\Omega^{2}\left(\operatorname{tr} K^{2}+2 \kappa \omega\right) \cos (\Omega \tau)\right. \\
& \left.+\Omega \operatorname{tr} K\left(\kappa \omega-2 \Omega^{2} \operatorname{tr} K\right) \sin (\Omega \tau)\right] .
\end{aligned}
$$

At the Pyragas points $\left(\Omega=\Omega^{A}, \tau=\tau_{n}^{A}\right)$ this gives

$$
\left.\operatorname{sgn} \operatorname{Re}\left(\partial_{\tau} \eta\right)\right|_{A}=\operatorname{sgn}(\kappa) .
$$

The crossing direction of the Hopf eigenvalues at the Hopf $B$ points $\left(\Omega=\Omega^{B}, \tau=\tau_{n}^{B}\right)$, on the other hand, is given by

$$
\begin{aligned}
\left.\operatorname{sgn} \operatorname{Re}\left(\partial_{\tau} \eta\right)\right|_{B} & =\operatorname{sgn}\left[2 \operatorname{tr} K^{2} \kappa^{2}-\operatorname{tr} K^{2} \kappa \omega-\kappa \omega^{3}\right] \\
& =-\operatorname{sgn}(\kappa) \cdot \operatorname{sgn}\left[\omega^{3}+\operatorname{tr} K^{2}(\omega-2 \kappa)\right] .
\end{aligned}
$$

For the allowed $\kappa$ values $(\kappa<\omega / 2)$ the second term is positive, and this expression reduces to

$$
\operatorname{sgn} \operatorname{Re}\left(\partial_{\tau} \eta\right)=-\operatorname{sgn}(\kappa),
$$

and hence the crossing direction is opposite to that at the Pyragas points.

(3) Slope of Hopf and Pyragas curve: By implicit differentiation of the characteristic equation (5) with respect to $\mu$, we find the slope of the Hopf curve at the Pyragas points

$$
\left.\frac{\partial}{\partial \mu} \tau_{H}\right|_{\tau=\tau_{n}^{A}}=-2 \frac{d(n \pi \operatorname{tr} K+\omega)+c n \pi \kappa}{\omega^{2} \kappa} .
$$

The slope of the Pyragas curve at $\mu=0$ is given by

$$
\frac{\partial}{\partial \mu} \tau_{P}=-\frac{2 \pi n}{\omega^{2}}\left(c-\frac{b d}{a}\right) .
$$

For the IP case the period of the orbit increases with increasing distance from the bifurcation, and the Pyragas curve emanates to the upper left from the Pyragas point. This is the situation depicted in Fig. 4. For stabilization we need a (2)-region above and a (0)-region below the $n$th Pyragas point. This means the eigenvalue crossing directions has to be positive at the $A$ points and negative at the $B$ points, i.e.,

$$
\kappa>0 .
$$

The Pyragas curve has to lie above the Hopf curve for $\mu<0$, which means the slopes at $\mu=0$ have to obey $\partial_{\mu} \tau_{p}<\partial_{\mu} \tau_{H}$, which gives

$$
\operatorname{tr} K<-\frac{b}{a} \kappa-\frac{\omega}{\pi n} .
$$

Finally the ordering of the Hopf points has to be $\tau_{n-1}^{B} \leqslant \tau_{n}^{A}$. Inserting the calculated $\tau$ values [see Eq. (8)] gives two cases.

(1) For $\operatorname{tr} K \geqslant 0$ inserting the $\tau$ values gives

$$
\varphi \leqslant 2 \pi+2 \pi n\left(\frac{\Omega^{B}}{\omega}-1\right) .
$$

Depending on the values of $n, \kappa$, and $\omega$ the right-hand side may be negative, and the inequality can not be fulfilled since $\varphi \in[0, \pi]$. Stabilization is possible only if the right-hand side is positive, i.e., if

$$
\kappa \leqslant \omega \frac{2 n-1}{2 n^{2}} .
$$

Note that for $n=1$ this coincides with our initial condition $\kappa<\omega / 2$. For this valid $\kappa$-range, inserting $\varphi$ from Eq. (9), the inequality (13) gives a condition on the magnitude of $\operatorname{tr} K$

$$
\operatorname{tr} K \geqslant \frac{\kappa \omega}{\Omega^{B}} \cot (\pi n \sqrt{1-2 \kappa / \omega}) .
$$

(2) For $\operatorname{tr} K<0$ we find

$$
\varphi \geqslant 2 \pi n\left(1-\frac{\Omega^{B}}{\omega}\right) .
$$


Inserting $\varphi$ then gives the same bound as above:

$$
\operatorname{tr} K \geqslant \frac{\kappa \omega}{\Omega^{B}} \cot (\pi n \sqrt{1-2 \kappa / \omega}) ;
$$

however, in this case the $\kappa$ domain is different, and the left-hand side as well as the right-hand side are negative.

The condition for the Hopf point ordering, for the IP case (with $\kappa>0$ ), can thus be summarized by

$$
\begin{gathered}
\kappa \leqslant \omega \frac{2 n-1}{2 n^{2}}, \\
\operatorname{tr} K \geqslant \frac{\kappa \omega}{\sqrt{\omega^{2}-2 \kappa \omega}} \cot (\pi n \sqrt{1-2 \kappa / \omega}) .
\end{gathered}
$$

For the DP case the period of the orbit decreases with increasing distance from the bifurcation, and the Pyragas curve emanates to the lower left from the Pyragas point. For stabilization we need a (0)-region above and a (2)-region below the emanating Pyragas point. This means the eigenvalue crossing directions has to be negative at the $A$ points and positive at the $B$ points, i.e.,

$$
\kappa<0 \text {. }
$$

The Pyragas curve has to lie below the Hopf curve for $\mu<0$, which means the slopes at $\mu=0$ have to obey $\partial_{\mu} \tau_{p}>\partial_{\mu} \tau_{H}$, which gives

$$
\operatorname{tr} K<-\frac{b}{a} \kappa-\frac{\omega}{n \pi} .
$$

This is the same as condition (12), which is no contradiction, since $\kappa$ has opposite sign. Finally the ordering of the Hopf points has to be $\tau_{n}^{B} \geqslant \tau_{n}^{A}$. A similar discussion as above shows that there can only be a solution if

$$
-\omega \frac{2 n+1}{2 n^{2}} \leqslant \kappa
$$

and that the boundary for $\operatorname{tr} K$ is the same as above:

$$
\operatorname{tr} K \geqslant \frac{\kappa \omega}{\sqrt{\omega^{2}-2 \kappa \omega}} \cot (\pi n \sqrt{1-2 \kappa / \omega}) .
$$

\section{STABILIZATION WITH SYMMETRIC FEEDBACK MATRICES}

We will now discuss a method to stabilize the UPO with symmetric feedback matrices $(\kappa=0)$. Consider the normal form model from above for $\kappa=0$, which was not controllable before, with an additional latency time $\delta$ in the feedback

$$
\begin{aligned}
\frac{d}{d t}\left(\begin{array}{l}
x \\
y
\end{array}\right)= & {\left[\begin{array}{cc}
d \mu+a r^{2} & -\left(\omega+c \mu+b r^{2}\right) \\
\omega+c \mu+b r^{2} & d \mu+a r^{2}
\end{array}\right]\left(\begin{array}{l}
x \\
y
\end{array}\right) } \\
& +\left[\begin{array}{cc}
\operatorname{tr} K & 0 \\
0 & 0
\end{array}\right]\left(\begin{array}{l}
x(t-\tau-\delta)-x(t-\delta) \\
y(t-\tau-\delta)-y(t-\delta)
\end{array}\right) .
\end{aligned}
$$

Proceeding as above we find the characteristic equation for the eigenvalues of the FP:

$$
\chi(\eta)=(d \mu-\eta)^{2}+\operatorname{tr} K(d \mu-\eta) F(\eta)+(\omega+c \mu)^{2},
$$

where in this case $F(\eta)=e^{-\eta(\tau+\delta)}-e^{-\eta \delta}$. Below we present and derive the stabilization conditions for this case. Effectively the additional lag time makes the other component $y$ observable.

\section{A. Stabilization conditions}

We again distinguish the IP and DP case and define

$$
m=\left\{\begin{array}{lll}
+1 & \text { for } \quad c-b d / a>0 & \text { (IP case) } \\
-1 & \text { for } \quad c-b d / a<0 & \text { (DP case) }
\end{array}\right.
$$

Close to the bifurcation (limit $\mu \rightarrow 0$ ), the domain of control is bounded in the $(\delta, \operatorname{tr} K)$ plane by

$$
\begin{gathered}
|\operatorname{tr} K| \delta<1 / \omega, \\
m \operatorname{tr} K \sin (\omega \delta)<0, \\
\frac{\omega}{\pi n}<m \operatorname{tr} K\left(\frac{b}{a} \sin (\omega \delta)-\cos (\omega \delta)\right), \\
|\operatorname{tr} K(\psi)|<\left|\frac{X(\psi)^{2}-\omega^{2}}{2 X(\psi) \sin \psi}\right| \text { for } \delta(\psi)=\psi / X(\psi),
\end{gathered}
$$

where the last equation describes the boundary in parametric form (parameter $\psi>0$ ) with

$$
X(\psi):=\frac{\omega}{n}\left(\left\lceil\frac{1}{\pi} \psi\right\rceil-\frac{1}{\pi} \psi-\frac{m+1}{2}\right)+\omega<\Omega^{B}
$$

and $\lceil\bullet\rceil$ denotes the ceiling function. Condition (19) is only sufficient so that the actual domain of control may be larger. The other conditions describe exact boundaries of the domain of control.

Figures 5 and 7 show the domain of control in the $(\delta, \operatorname{tr} K)$ plane for the two cases. Figure 6 depicts the actual domain of control for the IP case calculated with the continuation software KNUT.

\section{B. Derivation of stabilization results}

(1) Location of Hopf points: To find the Hopf points, we evaluate the real and imaginary part of $0=\chi(\eta)$ at $\mu=0$, $\eta=i \Omega$ :

$$
\begin{gathered}
0=-\Omega^{2}+\omega^{2}+\Omega \operatorname{tr} K[\sin (\Omega \delta)-\sin (\Omega \delta+\Omega \tau)], \\
0=\Omega \operatorname{tr} K[\cos (\Omega \delta)-\cos (\Omega \delta+\Omega \tau)] .
\end{gathered}
$$

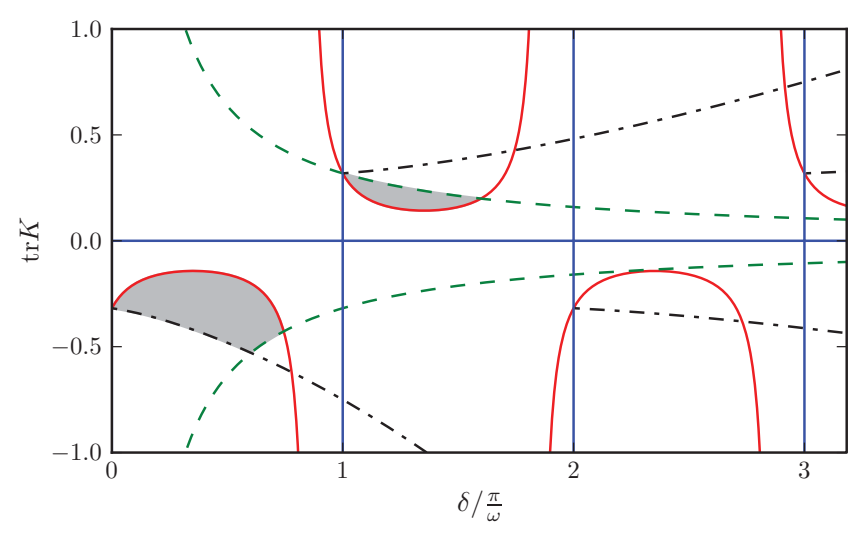

FIG. 5. (Color online) Control domain for the increasing period case in the $(\delta, \operatorname{tr} K)$ plane. The shaded regions depict the domain of control found analytically for the limit $\mu \rightarrow 0$. The curves and lines depict the boundaries given by the following inequalities: vertical and horizontal (blue) lines, (20); solid (red) curves, (21); dashed (green) curves, (19); dashed dotted (black) curves, Eq. (22). Parameters: $\omega=$ $1, b / a=-2, c=4, d=1, n=1$. 


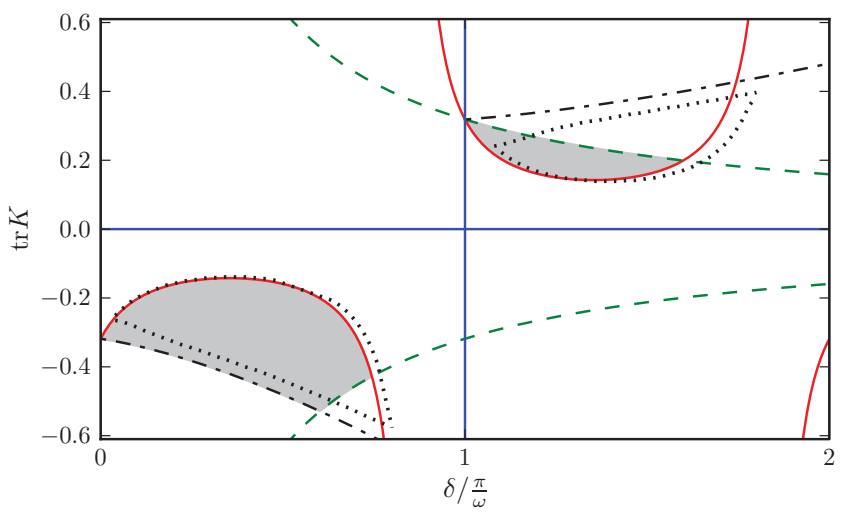

FIG. 6. (Color online) Blowup of Fig. 5. The dotted line depicts the domain of control for the periodic orbit calculated with KNUT. In comparison the shaded region depicts the domain found analytically for the limit $\mu \rightarrow 0$. Other curves as in Fig. 5. Note that the dashed (green) line, which corresponds to the sufficient condition (19), is not a necessary condition for the chosen parameters. The slight offset between the numerical and analytical control domains stems from the finite value of $\mu$ in the numerics. Parameters: $\omega=1, b / a=-2$, $c=4, d=1, n=1, \mu=0.005$.

The second equation yields

$$
\pm \Omega \delta+2 \pi n=\Omega \delta+\Omega \tau .
$$

The + sign gives the Hopf $A$ series:

$$
\tau_{n}^{A}=\frac{2 \pi n}{\omega}, \quad \Omega^{A}=\omega .
$$

The - sign gives the $B$ series:

$$
\tau_{n}^{B}=\frac{2 \pi n}{\Omega^{B}}-2 \delta,
$$

where $\Omega^{B}$ is left to be determined. Inserting this expression into Eq. (24a) gives a transcendental equation for $\Omega^{B}$ :

$$
0=f(\Omega):=\Omega^{2}-\omega^{2}-2 \operatorname{tr} K \Omega \sin (\Omega \delta) .
$$

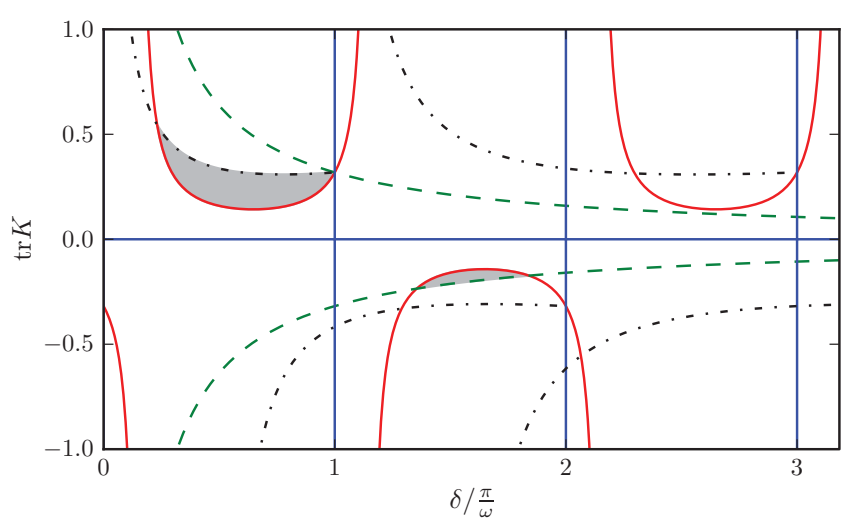

FIG. 7. (Color online) Control domain for the decreasing period case in the $(\delta, \operatorname{tr} K)$ plane. The shaded regions depict the domain of control found analytically for the limit $\mu \rightarrow 0$. The curves and lines depict the boundaries given by the following inequalities: vertical and horizontal (blue) lines, (20); solid (red) curves, (21); dashed (green) curves, (19); dashed dotted (black) curves, Eq. (22). Parameters: $\omega=$ $1, b / a=2, c=-4, d=1, n=1$.

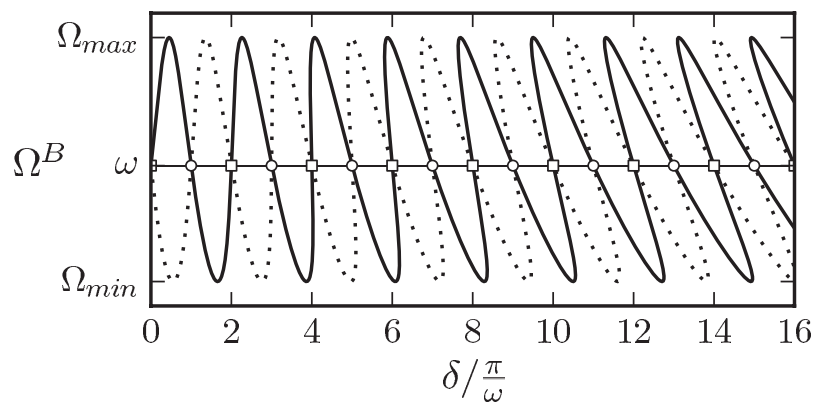

FIG. 8. (Color online) Solutions $\Omega^{B}$ of Eq. (25) vs latency $\delta$. The solid and dotted lines correspond to $\operatorname{tr} K=0.1>0$ and $\operatorname{tr} K=$ $-0.1<0$, respectively. Parameters: $\omega=1$.

Although we cannot explicitly obtain $\Omega^{B}(\delta)$, we can obtain a parametric representation. To do this we introduce a curve parameter $\psi=\Omega \delta$. Solving $0=f(\Omega)$ for $\Omega$ then gives the parametric solution:

$$
\begin{gathered}
\Omega^{B}=\operatorname{tr} K \sin \psi+\sqrt{\omega^{2}+\operatorname{tr} K^{2} \sin ^{2} \psi}, \\
\delta=\frac{\psi}{\Omega^{B}}=\frac{\psi}{\operatorname{tr} K \sin \psi+\sqrt{\omega^{2}+\operatorname{tr} K^{2} \sin ^{2} \psi}} .
\end{gathered}
$$

The $\Omega^{B}$ values lie in in the interval

$$
\begin{aligned}
\Omega^{B} & \in\left[\Omega_{\min }, \Omega_{\max }\right], \quad \text { with } \\
\Omega_{\min } & =-\operatorname{tr} K+\sqrt{\omega^{2}+\operatorname{tr} K^{2}}, \\
\Omega_{\max } & =\operatorname{tr} K+\sqrt{\omega^{2}+\operatorname{tr} K^{2}} .
\end{aligned}
$$

Figure 8 depicts the solutions $\Omega^{B}$ versus $\delta$. At the special points

$$
\delta_{k}^{*}=\frac{\pi}{\omega} k, \quad\left(k \in \mathbb{N}_{0}\right),
$$

$\Omega=\omega$ is always a solution of Eq. (25) and (some of) the Hopf $B$ points lie on the Pyragas points. Note that the labeling of Hopf $A$ and $B$ points is different in this case:

$$
\tau_{n}^{A}=\frac{2 \pi n}{\omega}=\frac{2 \pi(n+k)}{\omega}-2 \delta_{k}^{*}=\tau_{n+k}^{B} .
$$

For simplicity we will now consider the case where Eq. (25) has a single (positive) solution; i.e., we consider $\delta$ and $|\operatorname{tr} K|$ small enough such that the curve in Fig. 8 does not fold back.

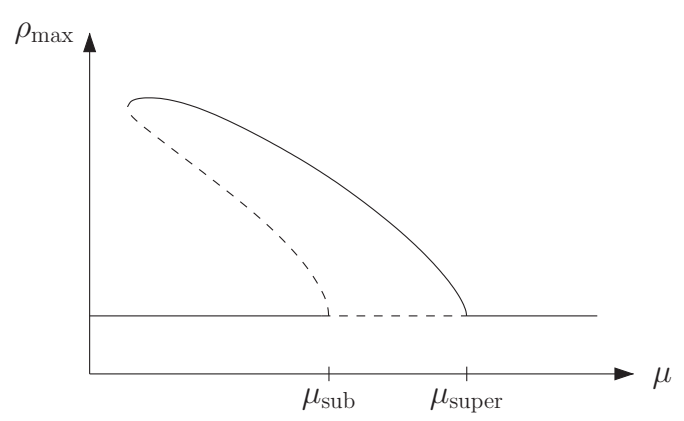

FIG. 9. Schematic bifurcation diagram of the laser. At $\mu_{\text {sub }}$ the lasing fixed point loses its stability in a subcritical Hopf bifurcation. The unstable periodic orbit born at this bifurcation is the target orbit. For a detailed discussion of this bifurcation scenario see Ref. [26]. 
Implicit differentiation of Eq. (25) with respect to $\delta$ gives the slope of the $\Omega^{B}$ curve at $\delta=\delta_{k}^{*}$ :

$$
\left.\partial_{\delta} \Omega^{B}\right|_{\delta=\delta_{k}^{*}}=\frac{\operatorname{tr} K \omega}{(-1)^{k}-\omega \delta_{k}^{*} \operatorname{tr} K} .
$$

We can find a sufficient condition that ensures a single solution by requiring that the signs of the slopes at the $\delta_{k}^{*}$ values alternate with $k$. This is the case if

$$
|\operatorname{tr} K| \delta<1 / \omega
$$

Note that since this condition is only sufficient, the corresponding curve need not be a boundary of the control domain; i.e., the domain of control may in fact be larger, as we will see below.

(2) Crossing direction of Hopf eigenvalue pair: To calculate the crossing direction of the Hopf eigenvalue pair, we differentiate Eq. (17) implicitly with respect to $\tau$. This expression evaluated at $\mu=0, \eta=i \Omega$ then gives the crossing direction:

$$
\begin{aligned}
\operatorname{sgnRe}\left(\partial_{\tau} \eta\right)= & \operatorname{sgn}\left[-\operatorname{tr} K^{2}+\operatorname{tr} K^{2} \cos (\Omega \tau)+\Omega \operatorname{tr} K^{2} \delta \sin (\Omega \tau)\right. \\
& -2 \operatorname{tr} K \Omega \sin (\Omega \tau+\Omega \delta)] .
\end{aligned}
$$

For the Hopf $A$ series this gives

$$
\left.\operatorname{sgnRe}\left(\partial_{\tau} \eta\right)\right|_{A}=-\operatorname{sgn}[\operatorname{tr} K \sin (\omega \delta)] .
$$

For the Hopf $B$ series we find

$$
\begin{aligned}
\left.\operatorname{sgnRe}\left(\partial_{\tau} \eta\right)\right|_{B}= & -\operatorname{sgn}\left[\operatorname{tr} K \sin \left(\Omega^{B} \delta\right)\right] \\
& \cdot \operatorname{sgn}\left[-\Omega+\Omega \operatorname{tr} K \delta \cos \left(\Omega^{B} \delta\right)+\operatorname{tr} K \sin \left(\Omega^{B} \delta\right)\right] \\
= & -\operatorname{sgn}\left[\operatorname{tr} K \sin \left(\Omega^{B} \delta\right)\right] \cdot \operatorname{sgn}\left[-f^{\prime}\left(\Omega^{B}\right)\right] \\
= & \operatorname{sgn}\left[\left(\Omega^{B}\right)^{2}-\omega^{2}\right] \cdot \operatorname{sgn} f^{\prime}\left(\Omega^{B}\right) .
\end{aligned}
$$

From Eq. (25) we find that $f(0)=-\omega^{2}$ and $\lim _{\Omega \rightarrow \infty} f(\Omega)=$ $\infty$, and because we consider the case of a single positive solution $0=f\left(\Omega^{B}\right)$ the slope $f^{\prime}\left(\Omega^{B}\right)$ is positive. The single solution $\Omega^{B}$ oscillates with increasing $\delta$ around $\omega$ (see Fig. 8) and is larger than $\omega$ if $\operatorname{tr} K>0$ and $\delta \in\left(\delta_{k}^{*}, \delta_{k+1}^{*}\right)$ with even $k$ or if $\operatorname{tr} K<0$ and $\delta \in\left(\delta_{k}^{*}, \delta_{k+1}^{*}\right)$ with odd $k$. Thus the crossing direction is in fact opposite to that of the $A$ series:

$$
\left.\operatorname{sgnRe}\left(\partial_{\tau} \eta\right)\right|_{B}=-\left.\operatorname{sgnRe}\left(\partial_{\tau} \eta\right)\right|_{A}=\operatorname{sgn}[\operatorname{tr} K \sin (\omega \delta)] .
$$

(3) Slope of Hopf and Pyragas curve: The slope of the Hopf curve at the Pyragas points can be calculated by implicit differentiation of the characteristic equation with respect to $\mu$. Evaluated at the Pyragas points we find

$$
\frac{\partial}{\partial \mu} \tau_{H}=\frac{-2 n \pi \operatorname{tr} K(c-d \cot (\omega \delta))+2 d \omega / \sin (\omega \delta)}{\operatorname{tr} K \omega^{2}} .
$$

Putting the pieces together: For $-(c-b d / a)<0$ the period of the orbit increases with increasing distance from the bifurcation, and the Pyragas curve emanates to the upper left from the Pyragas point. For stabilization we need a (2)-region above and a (0)-region below the $n$th Pyragas point. This means the eigenvalue crossing direction has to be positive at the $A$ points and negative at the $B$ points, i.e.,

$$
\operatorname{tr} K \sin (\omega \delta)<0 \text {. }
$$

The Pyragas curve has to lie above the Hopf curve for $\mu<0$, i.e., the slopes at $\mu=0$ have to obey $\partial_{\mu} \tau_{p}<\partial_{\mu} \tau_{H}$. This gives

$$
\frac{b}{a}<\cot (\omega \delta)+\frac{\omega}{\pi n \operatorname{tr} K \sin (\omega \delta)},
$$

which can be written as

$$
\operatorname{tr} K\left(\frac{b}{a} \sin (\omega \delta)-\cos (\omega \delta)\right)>\frac{\omega}{\pi n},
$$

taking into account Eq. (31).

There is at most one Hopf $B$ point between two successive $A$ points, because

$$
\Delta \tau^{B}=\frac{2 \pi}{\Omega^{B}}>\frac{2 \pi}{\omega}=\Delta \tau^{A} .
$$

To have a (0)-region below the Pyragas point, there has to be exactly one such point in between, to compensate for the increase in the number of unstable dimensions at the $A$ points. The Hopf $B$ points start at $\tau_{0}^{B}=-2 \delta$, and thus the first $B$ point with positive $\tau^{B}$ is given by $\tau_{\tilde{k}}^{B}$, where $\tilde{k}$ is the smallest integer with

$$
\tilde{k} \cdot \Delta \tau^{B}>2 \delta
$$

i. e.,

$$
\tilde{k}=\left\lceil\frac{2 \delta}{\Delta \tau^{B}}\right\rceil=\left\lceil\delta \frac{\Omega^{B}}{\pi}\right\rceil .
$$

With this index the Hopf point ordering can be written as

$$
\tau_{\tilde{k}}^{B}<\tau_{1}^{A}<\tau_{\tilde{k}+1}^{B}<\tau_{2}^{A}<\cdots<\tau_{\tilde{k}+n-1}^{B}<\tau_{n}^{A} .
$$

It is sufficient to require $\tau_{\tilde{k}+n-1}^{B}<\tau_{n}^{A}$ because this condition is the strictest. Thus we have

$$
\left\lceil\delta \frac{\Omega^{B}}{\pi}\right\rceil-\delta \frac{\Omega^{B}}{\pi}-1<n\left(\frac{\Omega^{B}}{\omega}-1\right) .
$$

Using the parametric representation with $\psi=\Omega^{B} \delta$ [Eq. (26)] gives

$$
X(\psi):=\frac{\omega}{n}\left(\left\lceil\frac{1}{\pi} \psi\right\rceil-\frac{1}{\pi} \psi-1\right)+\omega<\Omega^{B} .
$$

Inserting $\Omega^{B}(\psi, \operatorname{tr} K)$ from Eq. (26) and solving for $\operatorname{tr} K$ then gives the boundary curve in parametric form

$$
\begin{gathered}
|\operatorname{tr} K(\psi)|<\left|\frac{X(\psi)^{2}-\omega^{2}}{2 X(\psi) \sin \psi}\right|, \\
\delta(\psi)=\frac{\psi}{X(\psi)} .
\end{gathered}
$$

For $-(c-b d / a)>0$ the period of the orbit decreases with increasing distance from the bifurcation, and the Pyragas curve emanates to the lower left from the Pyragas point. For stabilization we need a (0)-region above and a (2)-region below the emanating Pyragas point. This means the eigenvalue crossing directions have to be negative at the $A$ points and positive at the $B$ points, i.e.,

$$
\operatorname{tr} K \sin (\omega \delta)>0
$$


The Pyragas curve has to lie below the Hopf curve for $\mu<0$; i.e., the slopes at $\mu=0$ have to obey $\partial_{\mu} \tau_{p}>\partial_{\mu} \tau_{H}$, which gives

$$
\frac{b}{a}>\cot (\omega \delta)+\frac{\omega}{\pi n \operatorname{tr} K \sin (\omega \delta)} .
$$

This finally gives the same condition as in the IP case [Eq. (32)]:

$$
\operatorname{tr} K\left(\frac{b}{a} \sin (\omega \delta)-\cos (\omega \delta)\right)>\frac{\omega}{\pi n} .
$$

There is at least one Hopf $B$ point between two successive $A$ points, because

$$
\Delta \tau^{B}=\frac{2 \pi}{\Omega^{B}}<\frac{2 \pi}{\omega}=\Delta \tau^{A} .
$$

To have a (2)-region below the Pyragas point, there has to be exactly one such point in between. The Hopf $B$ points start at $\tau_{0}^{B}=-2 \delta$, and again the first $B$ point with positive $\tau^{B}$ is given by $\tau_{\tilde{k}}^{B}$, where $\tilde{k}$ is given as above by

$$
\tilde{k}=\left\lceil\frac{2 \delta}{\Delta \tau^{B}}\right\rceil=\left\lceil\delta \frac{\Omega^{B}}{\pi}\right\rceil .
$$

The Hopf point ordering is then given by

$$
\tau_{\tilde{k}}^{B}<\tau_{1}^{A}<\tau_{\tilde{k}+1}^{B}<\tau_{2}^{A}<\cdots<\tau_{n}^{A}<\tau_{\tilde{k}+n}^{B} .
$$

It is sufficient to require $\tau_{n}^{A}<\tau_{\tilde{k}+n}^{B}$, which yields

$$
\left\lceil\delta \frac{\Omega^{B}}{\pi}\right\rceil-\delta \frac{\Omega^{B}}{\pi}>n\left(\frac{\Omega^{B}}{\omega}-1\right) .
$$

Using again the parametric representation [Eq. (26)] we obtain this time

$$
X(\psi):=\frac{\omega}{n}\left(\left\lceil\frac{1}{\pi} \psi\right\rceil-\frac{1}{\pi} \psi\right)+\omega>\Omega^{B},
$$

and with $\Omega^{B}(\psi, \operatorname{tr} K)$ from Eq. (26) the boundary curve in parametric form

$$
\begin{aligned}
|\operatorname{tr} K(\psi)| & <\left|\frac{X(\psi)^{2}-\omega^{2}}{2 X(\psi) \sin \psi}\right|, \\
\delta(\psi) & =\frac{\psi}{X(\psi)} .
\end{aligned}
$$

Note that since we consider only the case where Eq. (25) has a single solution [see Eq. (28)], all the conditions we constructed are sufficient but not necessary. The actual domains of control in Figs. 5 and 7 may in fact be larger.

\section{APPLICATIONS TO LASERS}

In the following we will discuss the stabilizing of a subcritical Hopf orbit in a laser using optoelectronic feedback. Consider the following dimensionless laser model [26-29] of a laser with a passive dispersive reflector:

$$
\begin{gathered}
\frac{d}{d t} \rho=N \rho, \\
T \frac{d}{d t} N=p-N-(1+N) k_{\mu}(N) \rho,
\end{gathered}
$$

where $N$ and $\rho$ are the inversion and photon number in dimensionless units, respectively, $T$ is the ratio between carrier and photon lifetime, and $p$ is the injection current in excess of the laser threshold.

The passive dispersive reflector is modeled by the function

$$
k_{\mu}(N)=K_{\mu}+\frac{A W^{2}}{4(N-\mu)^{2}+W^{2}},
$$

where $K_{\mu}$ is chosen such that $k_{\mu}(0)=1$ :

$$
K_{\mu}=1-\frac{A W^{2}}{4 \mu^{2}+W^{2}} .
$$

This laser model shows sub- and supercritical Hopf bifurcations [26] depending on the parameters.

Suppose now that there is a Hopf bifurcation at $\mu=\mu_{*}$. To apply the discussion from the last section, we need to bring the laser equations close to this Hopf bifurcation (small $\Delta \mu:=\mu-\mu_{*}$ ) into the normal form of Eq. (2):

$$
\frac{d}{d t}\left(\begin{array}{l}
x \\
y
\end{array}\right)=\left[\begin{array}{cc}
d \mu+a r^{2} & -\left(\omega+c \mu+b r^{2}\right) \\
\omega+c \mu+b r^{2} & d \mu+a r^{2}
\end{array}\right]\left(\begin{array}{l}
x \\
y
\end{array}\right)
$$

i.e., we need to find the coefficients $a, b, c$, and $d$.

Let us first discuss the location of the Hopf bifurcation. The Jacobian of the system is given by

$J=\left[\begin{array}{cc}N & \rho \\ -\frac{1}{T}(1+N) k_{\mu}(N) & -\frac{1}{T}\left[1+\rho k_{\mu}(N)+\rho(1+N) k_{\mu}^{\prime}(N)\right]\end{array}\right]$.

At the lasing $\operatorname{FP}(\rho, N)=(p, 0)$ the Jacobian is given by

$$
J=\left[\begin{array}{cc}
0 & p \\
-\frac{1}{T} & -\frac{1}{T}\left[1+p+p k_{\mu}^{\prime}(0)\right]
\end{array}\right],
$$

and the eigenvalues are

$$
\lambda_{ \pm}=-\gamma \pm i \sqrt{\omega^{2}-\gamma^{2}}
$$

with

$$
\gamma=\frac{1}{2 T}\left[1+p+p k_{\mu}^{\prime}(0)\right], \quad \omega=\sqrt{p / T} .
$$

The function $k_{\mu}$ depends on $\mu$, which we will treat as a bifurcation parameter. If for some value $\mu_{*}$

$$
k_{\mu_{*}}^{\prime}(0)=-\frac{1+p}{p},
$$

then the real part of the eigenvalues vanishes $(\gamma=0)$, and a Hopf bifurcation occurs. The values $\mu_{*}$ where this happens solve the implicit equation

$$
\frac{8 A W^{2} \mu_{*}}{\left(W^{2}+4 \mu_{*}^{2}\right)^{2}}=-\frac{1+p}{p} .
$$

The Jacobian then has the eigenvalues

$$
\lambda_{ \pm}= \pm i \omega .
$$

From these eigenvalues we can already determine two of the coefficients

$$
\begin{aligned}
d & =\left.\frac{\partial}{\partial \mu} \operatorname{Re}\left(\lambda_{ \pm}(\mu)\right)\right|_{\mu=\mu_{*}}=-\left.\frac{p}{2 T} \frac{\partial}{\partial \mu} k_{\mu}^{\prime}(0)\right|_{\mu=\mu_{*}} \\
& =\frac{4 p A W^{2}\left(12 \mu_{*}^{2}-W^{2}\right)}{T\left(4 \mu_{*}^{2}+W^{2}\right)^{2}}
\end{aligned}
$$


and

$$
\begin{aligned}
c & =\left.\frac{\partial}{\partial \mu} \operatorname{Im}\left(\lambda_{+}(\mu)\right)\right|_{\mu=\mu_{*}} \\
& =\left.\frac{\partial}{\partial \mu} \sqrt{\omega^{2}-\gamma^{2}}\right|_{\mu=\mu_{*}}=\left.\frac{-\gamma \frac{\partial}{\partial \mu} \gamma}{\sqrt{\omega^{2}-\gamma^{2}}}\right|_{\mu=\mu_{*}}=0,
\end{aligned}
$$

where we have used $\gamma=0$ at $\mu=\mu_{*}$ in the last equality.

To arrive at a normal form, we use the transformation

$$
U=\left[\begin{array}{cc}
p & p \\
\omega & -\omega
\end{array}\right], \quad U^{-1}=\left[\begin{array}{cc}
\frac{1}{2 p} & \frac{1}{2 \omega} \\
\frac{1}{2 p} & \frac{-1}{2 \omega}
\end{array}\right]
$$

to define the new coordinates $x$ and $y$ according to

$$
\left(\begin{array}{l}
x \\
y
\end{array}\right)=U^{-1}\left(\begin{array}{c}
\rho-p \\
N
\end{array}\right), \quad\left(\begin{array}{l}
\rho \\
N
\end{array}\right)=U\left(\begin{array}{l}
x \\
y
\end{array}\right)+\left(\begin{array}{l}
p \\
0
\end{array}\right) .
$$

In these new coordinates the dynamical equations are given by

$$
\frac{d}{d t}\left(\begin{array}{l}
x \\
y
\end{array}\right)=\left[\begin{array}{cc}
d \Delta \mu & -\omega \\
\omega & d \Delta \mu
\end{array}\right]\left(\begin{array}{l}
x \\
y
\end{array}\right)+\left(\begin{array}{l}
f(x, y) \\
g(x, y)
\end{array}\right),
$$

where $f$ and $g$ carry the nonlinear terms. We can then calculate the other two coefficients using the well-known expressions [30]

$$
\begin{aligned}
a= & \frac{1}{16}\left[f_{x x x}+f_{x y y}+g_{x x y}+g_{y y y}\right]+\frac{1}{16 \omega}\left[f_{x y}\left(f_{x x}+f_{y y}\right)\right. \\
& \left.-g_{x y}\left(g_{x x}+g_{y y}\right)-f_{x x} g_{x x}+f_{y y} g_{y y}\right] \\
= & \frac{2+\left(2-k_{\mu_{*}}^{(2)}\right) p-\left(3 k_{\mu_{*}}^{(2)}+k_{\mu_{*}}^{(3)}\right) p^{2}}{8 T^{2}},
\end{aligned}
$$

and

$$
\begin{aligned}
b= & \frac{1}{16}\left[g_{x x x}+g_{x y y}-f_{y y y}-f_{x y y}\right] \\
& +\frac{1}{48 \omega}\left[f_{x x} g_{x y}+f_{x y} g_{y y}-2\left(f_{x x}^{2}+f_{x y}^{2}+g_{x y}^{2}+2 g_{y y}^{2}\right)\right. \\
& \left.-5\left(f_{y y}^{2}+g_{x x}^{2}+f_{x x} f_{y y}+g_{x x} g_{y y}+f_{x y} g_{x x}-f_{y y} g_{x y}\right)\right] \\
= & -\frac{1}{12 \omega T^{3}}\left[\left(2-k_{\mu_{*}}^{(2)}\right)^{2} p^{3}+T+\left(2-k_{\mu_{*}}^{(2)}\right)(T+4) p^{2}\right. \\
& \left.+\left(T^{2}+2 T+4\right) p\right],
\end{aligned}
$$

where $f_{x y}=\left(\partial_{x} \partial_{y} f\right)(0,0)$, etc. Here, we have used the expansion

$$
k_{\mu_{*}}(N)=1-\frac{1+p}{p} N+\frac{k_{\mu_{*}}^{(2)}}{2 !} N^{2}+\frac{k_{\mu_{*}}^{(3)}}{3 !} N^{3} .
$$

We now consider a concrete example with the laser parameters shown in Table I. With these typical values of the parameters we can start to calculate the values of $\mu$ where Hopf bifurcations take place, according to Eq. (37). Solving

TABLE I. Parameters of the Hopf laser model.

\begin{tabular}{lcccc}
\hline \hline Parameter & $p$ & $A$ & $W$ & $T$ \\
\hline Value & 2.0 & 1.0 & 0.02 & 1000 \\
\hline \hline
\end{tabular}

TABLE II. Parameters of the normal form model describing the laser close to the subcritical Hopf bifurcation.

\begin{tabular}{lc}
\hline \hline Parameter & Value \\
\hline$a$ & $3.074 \times 10^{-3}$ \\
$b$ & $-3.214 \times 10^{-3}$ \\
$c$ & 0.0 \\
$d$ & $8.576 \times 10^{-2}$ \\
$\omega$ & $4.472 \times 10^{-2}$ \\
\hline \hline
\end{tabular}

this equation numerically we find two Hopf bifurcations

$$
\mu_{\mathrm{sub}} \approx-4.976 \times 10^{-2}, \quad \mu_{\text {super }} \approx-7.5 \times 10^{-4},
$$

where $\mu_{\text {sub }}$ is a subcritical bifurcation and $\mu_{\text {super }}$ is a supercritical bifurcation. Figure 9 depicts the basic bifurcation diagram. Using the laser parameters we can now calculate the Hopf normal form parameters at the subcritical bifurcation $\mu=\mu_{\text {sub }}$. The approximate values are given in Table II. From the signs of $a$ and $d$ we see that it is indeed a subcritical Hopf bifurcation with the stable FP lying to the left of the bifurcation point $\left(\mu<\mu_{\text {sub }}\right)$. Furthermore,

$$
-(c-b d / a) \approx-8.967 \times 10^{-2}<0
$$

implies that we have the IP case.

To stabilize the subcritical Hopf orbit we now consider delayed optoelectronic feedback of the form

$$
\begin{gathered}
\frac{d}{d t} \rho=N \rho, \\
T \frac{d}{d t} N=p+\sigma(\rho(t-\tau)-\rho(t))-N-(1+N) k_{\mu}(N) \rho,
\end{gathered}
$$

where the control signal is given by $\sigma\left(\rho_{\tau}-\rho\right)$. Optoelectronic feedback can be realized [31] by measuring the intensity of the laser with a photodiode and modulating the pump current according to the delayed difference signal.

To find parameters $\sigma$ for successful control we first need to understand what the control term will become after the normal form transformation. Since the normal form transformation leaves linear terms invariant [30], we need only to take the

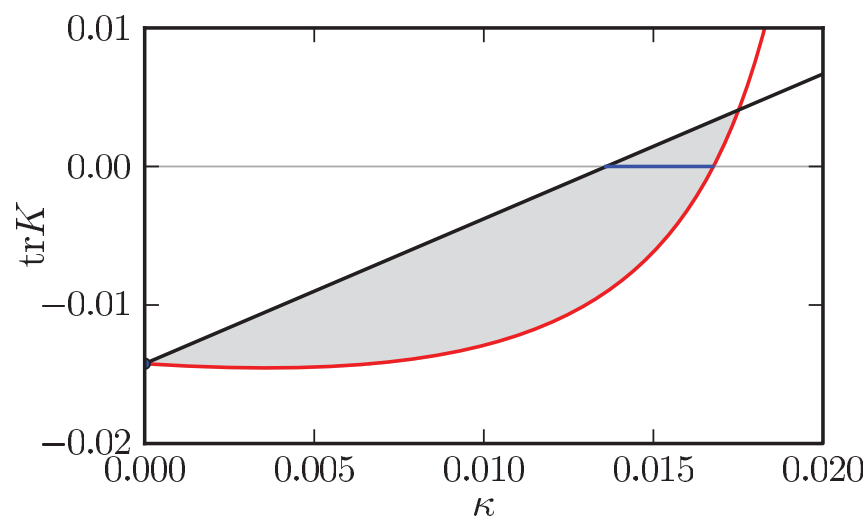

FIG. 10. (Color online) Domain of control for the Hopf normal form with coefficients as in Table II. The intersection of the shaded area with the $\operatorname{tr} K=0$ line is the control interval [Eq. (40)]. 

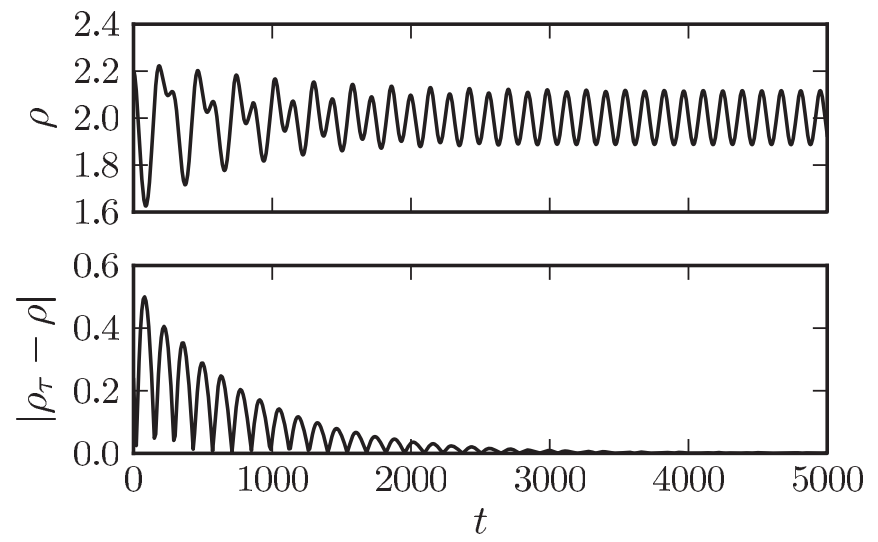

FIG. 11. Stabilization of the subcritical Hopf orbit in the laser system. Top panel: Time series of the intensity $\rho$. Bottom panel: Time series of the control signal. The laser parameters are as in Table I. The distance to the bifurcation is $\Delta \mu=\mu-\mu_{\text {sub }}=-0.001$, and the control amplitude is chosen as $\sigma=-0.3354$ in the control interval.

linear transformation Eq. (38) into account. This gives the control matrix in the normal form coordinates

$$
K=U^{-1}\left[\begin{array}{ll}
0 & 0 \\
\frac{\sigma}{T} & 0
\end{array}\right] \quad U=\left[\begin{array}{cc}
\frac{1}{2} \omega \sigma & \frac{1}{2} \omega \sigma \\
-\frac{1}{2} \omega \sigma & -\frac{1}{2} \omega \sigma
\end{array}\right]
$$

The relevant control parameters are then given by

$$
\kappa=-\omega \sigma, \quad \operatorname{tr} K=0 .
$$

On the other hand, we can find the domain of control in the $(\kappa, \operatorname{tr} K)$ plane from the calculated Hopf normal form coefficients as shown in Fig. 10. Using $\operatorname{tr} K=0$ we can calculate the control interval for $\kappa$ explicitly:

$$
\kappa \in\left[-\omega \frac{a}{\pi b}, \omega \frac{4 n^{2}-1}{8 n^{2}}\right] \approx[0.0136,0.0168]
$$

and the corresponding $\sigma$ interval

$$
\sigma \in\left[-\frac{4 n^{2}-1}{8 n^{2}}, \frac{a}{\pi b}\right] \approx[-0.375,-0.304]
$$

for $n=1$.

Figure 11 depicts the time series of the intensity and the control signal using a feedback gain in the control interval, which lead to successful stabilization of the subcritical periodic orbit.

A very similar feedback can also be realized all-optically by using polarization rotated optical feedback [32-35]. This is achieved by rotating the polarization axis of the emitted light by $\pi / 2$ into the perpendicular orientation and reinjecting this light into the laser. Due to the orthogonal polarization this injected signal does not contribute to the lasing mode but is still amplified and thus reduces the inversion. As a result one obtains a feedback of the following form:

$$
\begin{gathered}
\frac{d}{d t} \rho=N \rho \\
T \frac{d}{d t} N=p-N-(1+N) k_{\mu}(N)[\rho+\sigma \rho(t-\tau)] .
\end{gathered}
$$

Through interference of a delayed and a nondelayed light beam, it might then be possible to realize the Pyragas control scheme with this method:

$$
T \frac{d}{d t} N=p-N-(1+N) k_{\mu}(N)[\rho+\sigma(\rho(t-\tau)-\rho(t))] .
$$

The advantage would be that the control works even for very high oscillation frequencies beyond the bandwidth of electronic circuits.

Pyragas control has in the past been successfully applied experimentally. In most experimental implementations of optoelectronic Pyragas feedback, however, the overall latency in the control loop is very large. This makes successful optoelectronic control less feasible, as compared to all-optical control.

\section{SUMMARY}

In summary, we have introduced a class of Pyragas feedback schemes inspired by experimental conveniences and possibilities. We have constructed feedback parameters that allow for stabilization of odd-number orbits close to a subcritical Hopf bifurcation. We have proposed an extended control scheme using an additional latency time, which allows for stabilization with symmetric feedback matrices, which was impossible with previous feedback schemes. We demonstrated the successful control in both cases by numerical simulations and numerical continuation methods.

Furthermore, we showed how these control schemes can be applied to laser systems using optoelectronic feedback and how the constructed feedback parameters can be applied to this system by identifying the normal form parameters of the system close to the bifurcation.

\section{ACKNOWLEDGMENTS}

This work was supported by Deutsche Forschungsgemeinschaft in the framework of SFB 910: "Control of selforganizing nonlinear systems: Theoretical methods and concepts of application." We acknowledge stimulating discussions with Bernold Fiedler and Philipp Hövel.
[1] K. Pyragas, Phys. Lett. A 170, 421 (1992).

[2] C. von Loewenich, H. Benner, and W. Just, Phys. Rev. Lett. 93, 174101 (2004).

[3] E. Schöll and H. G. Schuster (eds.), Handbook of Chaos Control, 2nd ed. (Wiley-VCH, Weinheim, 2008).

[4] W. Just, A. Pelster, M. Schanz, and E. Schöll (eds.), Phil. Trans. R. Soc. A 368,301 (2010).
[5] V. Flunkert, S. Yanchuk, T. Dahms, and E. Schöll, Phys. Rev. Lett. 105, 254101 (2010).

[6] S. Schikora, P. Hövel, H. J. Wünsche, E. Schöll, and F. Henneberger, Phys. Rev. Lett. 97, 213902 (2006).

[7] J. Sieber, A. Gonzalez-Buelga, S. Neild, D. Wagg, and B. Krauskopf, Phys. Rev. Lett. 100, 244101 (2008).

[8] H. Nakajima, Phys. Lett. A 232, 207 (1997). 
[9] W. Just, T. Bernard, M. Ostheimer, E. Reibold, and H. Benner, Phys. Rev. Lett. 78, 203 (1997).

[10] I. Harrington and J. E. S. Socolar, Phys. Rev. E 64, 056206 (2001).

[11] K. Pyragas, V. Pyragas, and H. Benner, Phys. Rev. E 70, 056222 (2004).

[12] V. Pyragas and K. Pyragas, Phys. Rev. E 73, 036215 (2006).

[13] B. Fiedler, V. Flunkert, M. Georgi, P. Hövel, and E. Schöll, Phys. Rev. Lett. 98, 114101 (2007).

[14] C.-U. Choe, V. Flunkert, P. Hövel, H. Benner, and E. Schöll, Phys. Rev. E 75, 046206 (2007).

[15] W. Just, B. Fiedler, V. Flunkert, M. Georgi, P. Hövel, and E. Schöll, Phys. Rev. E 76, 026210 (2007).

[16] C. M. Postlethwaite and M. Silber, Phys. Rev. E 76, 056214 (2007).

[17] B. Fiedler, V. Flunkert, M. Georgi, P. Hövel, and E. Schöll, in Reviews of Nonlinear Dynamics and Complexity, edited by H. G. Schuster (Wiley-VCH, Weinheim, 2008), vol. 1, p. 53.

[18] B. Fiedler, S. Yanchuk, V. Flunkert, P. Hövel, H. J. Wünsche, and E. Schöll, Phys. Rev. E 77, 066207 (2008).

[19] B. Fiedler, Proc. 6th EUROMECH Nonlinear Dynamics Conference (ENOC-2008), edited by A. Fradkov and B. Andrievsky (2008), [http://lib.physcon.ru/?item=1568].

[20] M. Kehrt, P. Hövel, V. Flunkert, M. A. Dahlem, P. Rodin, and E. Schöll, Eur. Phys. J. B 68, 557 (2009).

[21] B. Fiedler, V. Flunkert, P. Hövel, and E. Schöll, Phil. Trans. R. Soc. London A 368, 319 (2010).
[22] G. Brown, C. M. Postlethwaite, and M. Silber, Physica D 240, 859 (2011).

[23] C. von Loewenich, H. Benner, and W. Just, Phys. Rev. E 82, 036204 (2010).

[24] S. Schikora, H. J. Wünsche, and F. Henneberger, Phys. Rev. E 83, 026203 (2011).

[25] R. Szalai, [http://seis.bris.ac.uk/rs1909/pdde/].

[26] V. Z. Tronciu, H. J. Wünsche, J. Sieber, K. Schneider, and F. Henneberger, Opt. Commun. 182, 221 (2000).

[27] V. Flunkert and E. Schöll, Phys. Rev. E 76, 066202 (2007).

[28] T. Dahms, P. Hövel, and E. Schöll, Phys. Rev. E 78, 056213 (2008).

[29] T. Dahms, V. Flunkert, F. Henneberger, P. Hövel, S. Schikora, E. Schöll, and H. J. Wünsche, Eur. Phys. J. ST 191, 71 (2010).

[30] J. Guckenheimer and P. Holmes, Nonlinear Oscillations, Dynamical Systems, and Bifurcations of Vector Fields (Springer, Berlin, 1986).

[31] H. D. I. Abarbanel, M. B. Kennel, L. Illing, S. Tang, H. F. Chen, and J. M. Liu, IEEE J. Quantum Electron. 37, 1301 (2001).

[32] K. Otsuka and J. L. Chern, Opt. Lett. 16, 1759 (1991).

[33] J. Houlihan, G. Huyet, and J. McInerney, Opt. Commun. 199, 175 (2001).

[34] F. Rogister, A. Locquet, D. Pieroux, M. Sciamanna, O. Deparis, P. Megret, and M. Blondel, Opt. Lett. 26, 1486 (2001).

[35] J. M. S. Solorio, D. W. Sukow, D. R. Hicks, and A. Gavrielides, Opt. Commun. 214, 327 (2002). 